\title{
Broadband Fourier-Domain Mode-Locked Lasers
}

\author{
Kevin HSU ${ }^{1}$, Panomsak MEEMON ${ }^{2,3}$, Kye-Sung LEE $^{3}$, Peter J. DELFYETT ${ }^{2}$, and \\ Jannick P. ROLLAND ${ }^{3,2}$
}

\author{
${ }^{1}$ Micron Optics, Inc., Atlanta, Georgia 30345, USA \\ ${ }^{2}$ CREOL, The College of Optics and Photonics, University of Central Florida, Orlando, Florida 32816, USA \\ ${ }^{3}$ The Institute of Optics, University of Rochester, Rochester, New York 14627, USA \\ *Corresponding author: Kevin HSU_Ｅ-mail:khsu@micronoptics.com
}

\begin{abstract}
Broadband, high-speed wavelength-swept lasers can substantially enhance applications in optical coherence tomography, chemical spectroscopy, and fiber-optic sensing. We report the demonstration of Fourier-domain mode-lock lasers operating at about $90 \mathrm{kHz}$ effective sweep rate over a $158 \mathrm{~nm}$ sweep range using a single-band design and over a $284 \mathrm{~nm}$ sweep range across the 1.3 $\mu \mathrm{m}$ to $1.5 \mu \mathrm{m}$ wavelength spectrum using a unique broadband design. A novel dual-detection full-range Fourier-domain optical coherence tomography system is developed which provides $7 \mu \mathrm{m}$ axial resolution (in air) at about $90 \mathrm{kHz}$ axial scan rate for mirror-image resolved Doppler imaging in a human finger and an African frog tadpole.
\end{abstract}

Keywords: Fourier-domain mode-locked laser, swept laser, tunable laser

\section{Introduction}

High-speed wavelength-swept laser technology offers versatile applications in optical frequency domain imaging (OFDI) [1-3], bio-chemical spectroscopy [4], and fiber-optic sensing [5]. Particularly in recent years, swept laser technology has been critical in advancing OFDI applications in the field of biomedical imaging (i.e., optical coherence tomography) [6-8] and industrial imaging, imparting the advantages of real-time in vivo diagnostics and high sensitivity. This technology's broader reach can be seen in its applications in dynamic absorption spectroscopy and chromatic confocal microscopy. Two general classes of swept laser have been driving the advance in the aforementioned applications:

One is continuous-wave (CW) swept laser system [1, 2] and the other is Fourier domain mode-locked (FDML) laser system demonstrated recently by Huber et al. [6].

The typical configuration of a $\mathrm{CW}$ swept laser incorporates a semiconductor optical amplifier (SOA), a high-performance fiber Fabry-Perot tunable filter (FFP-TF), and the associated isolators and couplers to form a unidirectional fiber ring laser (Fig. 1 (without fiber coil)). This general design embodies the collective advantages of SOA broad gain-bandwidth with direct modulation capability, FFP-TF wide tuning ranges (i.e., free spectral range $($ FSR $)>200 \mathrm{~nm})$, high finesse $(1,000$ to 20,000$)$, low loss $(<2 \mathrm{~dB})$, and fast scan rates. This class of swept laser system operates at arbitrary sweep rates, thus bears no relation to laser cavity resonance frequency and exhibits the following unique features: 1) Speed limitation: In cavity-tuning the maximum tuning speed is usually limited by the time-constant of the laser to build up lasing from the amplified

Received: 3 September 2010 / Revised version: 21 September 2010

(C) The Authors(s) 2010.This article is published with open access at Springerlink.com 
stimulated emission (ASE) background. This depends on filter function, ASE intensity, saturation power, laser gain, and cavity roundtrip time [2]. Initially the output power decreases gradually with the increasing scan rates. As the scan speed increases beyond the point for the amplifier to reach saturation, the output spectrum will begin to degrade rapidly. Ultimately, at excessive high scan frequencies, the light on average makes only one pass from SOA to filter and the cavity operates like a scanned incoherent source. Because lasing is built up repeatedly from ASE as the filter is tuned in wavelength, the filter bandwidth and cavity length must be optimized for specific high-speed applications. 2) Dynamic linewidth: Contrary to static linewidth, which depends strongly on the homogeneity of the gain medium, the dynamic linewidth is affected by the sweeping speed and constrained by filter bandwidth. 3) Spectral asymmetry: There is a strong spectral asymmetry between forward scans (from short to long wavelengths) and backward scans (from long to short wavelengths). This is caused by frequency downshift through intraband four-wave mixing within the SOA [9]. Scanning the filter in the same direction (forward scan) as the frequency shift allows intracavity light to experience less loss and enables higher lasing power and higher tuning speed operation. In general, a practical sweeping rate limit for CW swept lasers is in the range of about $20 \mathrm{kHz}$.

To overcome the speed limitations imposed by CW swept laser designs, a revolutionary FDML technique was demonstrated in $1310 \mathrm{~nm}$ and $1060 \mathrm{~nm}$ spectral regions [6, 7]. As illustrated in Fig. 1, this laser configuration is similar to standard ring-cavity design, except that FFP-TF is tuned in harmonic resonance to the fundamental cavity resonance that has been reduced by adding an additional length of fiber delay loop to match suitable electro-mechanical scan rates of FFP-TF. Rather than relying on repetitive transient built-up from ASE while the filter is tuned in wavelength, the SOA is seeded by the light from the previous frequency sweep with respect to individual frequency sweep components.

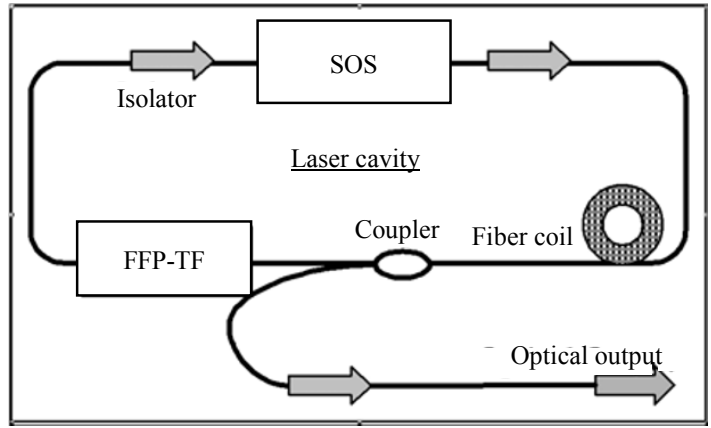

Fig. 1 Schematic of a single-band FDML laser module. The ring-cavity consists of a SOA as the gain medium, a FFP-TF for wavelength tuning, a fiber coil to set the cavity resonance frequency, isolators to enforce unidirectional lasing, and a fiber coupler for output coupling.

The FDML swept lasers have the advantage of high-speed, narrow linewidth, and high phase stability, and extensive effort has been invested in increasing sweep bandwidth and speed. In regard to sweep bandwidth, current FDML lasers using a single SOA have limited full-width-half-maximum (FWHM) sweep range of less than $117 \mathrm{~nm}$ for OFDI applications [8]. One approach to broaden the sweep range by combining two SOAs in parallel was first demonstrated in $1.3 \mu \mathrm{m}$ wavelength band in $\mathrm{CW}$ swept laser mode using a polygon scanner at $20 \mathrm{kHz}$ sweep frequency [10], which yielded a FWHM of $132 \mathrm{~nm}$ over $145 \mathrm{~nm}$ full range. Later a similar attempt in a FDML laser was demonstrated to provide slightly wider FWHM of $136.5 \mathrm{~nm}$ over $160 \mathrm{~nm}$ full range at much higher sweep frequency of $91.2 \mathrm{kHz}$ (bilateral) [11]. However, because two SOAs have substantially overlapped in gain spectra, this design requires using two 3-dB couplers to combine two SOAs, adding significant cavity loss which compromises lasing range and output power.

In this work, we first demonstrate that a FDML laser of FWHM of $158 \mathrm{~nm}$ can be achieved by using a single-band design. Next, by using a broadband design, the feasibility of a $284 \mathrm{~nm}$ sweep range across $1.3 \mu \mathrm{m}$ to $1.5 \mu \mathrm{m}$ dual wavelength spectra is demonstrated. 


\section{Single-band FDML}

By using a combination of high performance components to form single-band FDML laser as illustrated in Fig. 1, we have been able to obtain a FWHM sweep range as wide as about $158 \mathrm{~nm}$ (from $1252 \mathrm{~nm}$ to $1410 \mathrm{~nm}$ ) at an effective sweep rate (bilateral) of $89.2 \mathrm{kHz}$ as shown in Fig. 2. Note that due to the sinusoidal drive waveform, the temporal sweep profile is distorted accordingly, with most of the spectral range compressed in the middle of the sweep profile. In this arrangement, the FFP-TF has a FSR of about $160 \mathrm{~nm}$, a bandwidth of about $0.2 \mathrm{~nm}$, and a loss of less than $2 \mathrm{~dB}$. The current FFP-TF design has a first-order electro-mechanical resonance near $45 \mathrm{kHz}$ to $50 \mathrm{kHz}$. Therefore the total fiber cavity length (including fiber delay loop (Corning SMA28-e)) was designed for length of $4500 \mathrm{~m}$. The SOA has a peak gain of $32.9 \mathrm{~dB}$ at $1304 \mathrm{~nm}$, a bandwidth of about $46 \mathrm{~nm}$, and a $3-\mathrm{dB}$ saturation power Psat-3dB of $12.4 \mathrm{dBm}$. The output-coupling ratio of fused coupler is $20 \%$, which provides an average output power of $5.6 \mathrm{~mW}$.

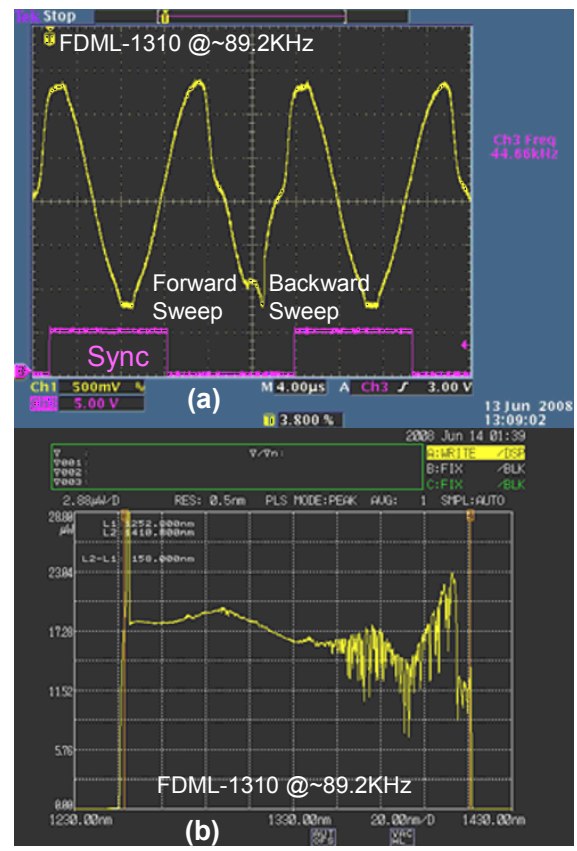

Fig. 2 Single-band $1310 \mathrm{~nm}$ FDML laser performance: (a) temporal swept laser profile at $89.2 \mathrm{kHz}$ double-sided sweep rate and (b) swept optical spectrum showing a FWHM sweep range of $158 \mathrm{~nm}$ (from $1252.0 \mathrm{~nm}$ to $1410.0 \mathrm{~nm}$ ).

\section{Broadband FDML}

To achieve broadband FDML, we chose to concatenate the adjacent spectral regions $(1.3 \mu \mathrm{m}$ and $1.5 \mu \mathrm{m}$ ) in a parallel configuration as illustrated in Fig. 3. The design consists of SOAs and isolators of adjacent bands arranged in a parallel-configuration, which is in turn coupled through two wavelength division multiplexers (WDMs) to complete the broadband ring-cavity. Two main technical challenges here are the ultra broadband component design and the requirements for bridging the dual spectra. The critical broadband components include a unique FFP-TF that provides FSR of $336.6 \mathrm{~nm}$, bandwidth of $0.2 \mathrm{~nm}$, loss of $1.5 \mathrm{~dB}$, and a fiber coupler for $20 \%$ output coupling. A fiber delay loop of $4500 \mathrm{~m}$ is included to set the cavity resonance frequency to $45 \mathrm{kHz}$. In addition to the challenges of maximizing the sweep range, the overlap region must be optimized to minimize discontinuity and the interference arising from equivalent Mach-Zehnder interferometric configuration of parallel SOA paths. In this regard, the SOA gain spectra and the WDM crossover region must be well matched. In our current work, two SOAs have the following parameters: $\mathrm{SOA}_{1.3}$ : peak $\sim 1299 \mathrm{~nm}$, gain $\sim 32.6 \mathrm{~dB}$, bandwidth $\sim 46.2 \mathrm{~nm}$, and Psat-3dB $\sim 12.4 \mathrm{dBm} ; \mathrm{SOA}_{1.5}$ : peak $\sim 1421 \mathrm{~nm}$, gain $\sim 21.8 \mathrm{~dB}$, bandwidth $\sim 61.7 \mathrm{~nm}$, and Psat- $3 \mathrm{~dB} \sim 13 \mathrm{dBm}$.

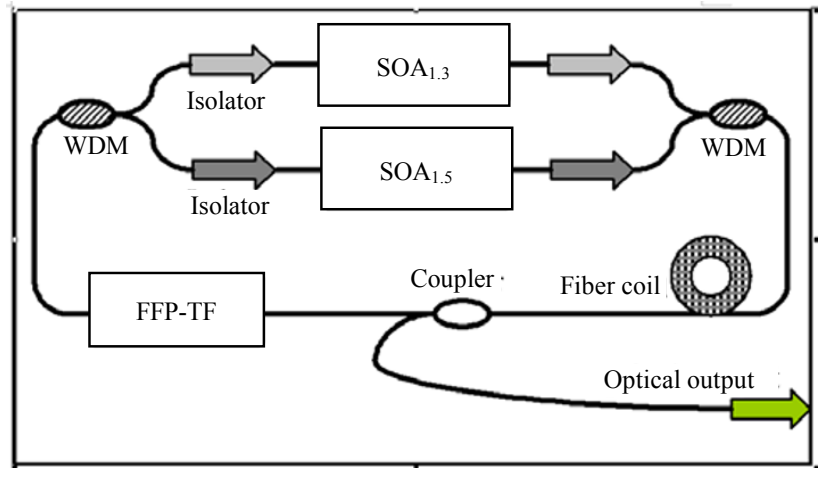

Fig. 3 Schematic of a broadband FDML module (the design consists of SOAs and isolators of adjacent bands arranged in a parallel configuration, then coupled through two WDMs to complete the ring-cavity, and the broadband components include a FFP-TF for wavelength tuning, a fiber coil to set the cavity resonance frequency, and a fiber coupler for output coupling). 
Tested separately in single-band CW swept laser mode, $1.3 \mu \mathrm{m}$ and $1.5 \mu \mathrm{m}$ SOAs can respectively provide lasing from $1240 \mathrm{~nm}$ to $1403 \mathrm{~nm}$ and from $1374 \mathrm{~nm}$ to $1538 \mathrm{~nm}$, indicating a substantial overlap region around $1400 \mathrm{~nm}$. The custom-fabricated WDM has a cross-over point centered at $1400 \mathrm{~nm}$ and an isolation level of $2 \mathrm{~dB}-$ $3 \mathrm{~dB}$, as illustrated in Fig. 4. This low isolation is important to minimize the spectral gap bridging two adjacent bands.

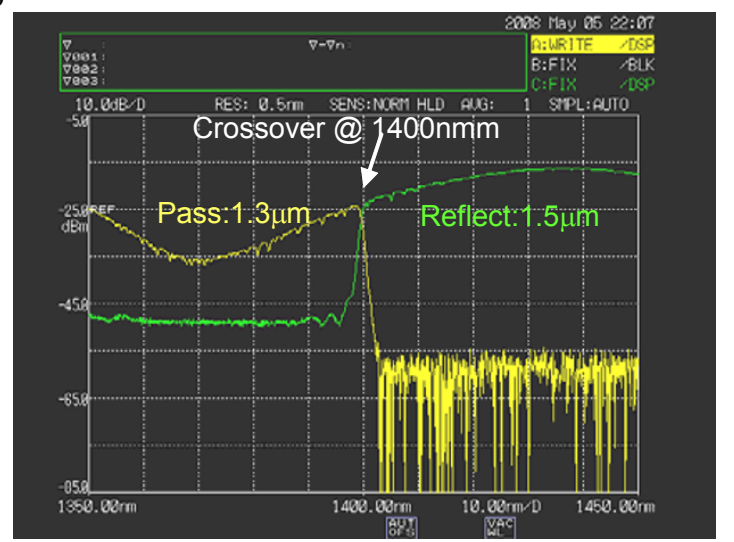

Fig. 4 Spectral characteristics of the WDM, having a band cross-over point centered at $1400 \mathrm{~nm}$ and an extinction of $2 \mathrm{~dB}$.

An initial test of parallel configuration in $\mathrm{CW}$ sweep mode at $100 \mathrm{~Hz}$ drive frequency without fiber delay loop shows a sweep range from $1251 \mathrm{~nm}$ to $1534 \mathrm{~nm}$, an average power $P_{a}$ of $6.48 \mathrm{~mW}$, and a discontinuity of about $5 \mathrm{~dB}$ as shown in Fig. 5 . Under broadband FDML operation driven at $44.5 \mathrm{kHz}$ sinusoidal frequency, an ultra broadband spectrum at about $89 \mathrm{kHz}$ bilateral sweep frequency is generated over a sweep range from $1252.8 \mathrm{~nm}$ to $1396.8 \mathrm{~nm}$ and from $1400 \mathrm{~nm}$ to $1536.8 \mathrm{~nm}$, with an average output power of $3.5 \mathrm{~mW}$. As demonstrated in Fig. 6, in spite of $4 \mathrm{~nm}$ spectral gap and 20-dB discontinuity, high-speed FDML operation can be obtained over a total range of about $284 \mathrm{~nm}$ and fiber dispersion over such a large range is not a limiting factor. The additional loss in fiber delay loop is one significant cause of the crossover gap and we expect that this can be improved by fusion splicing to all fiber connectors.

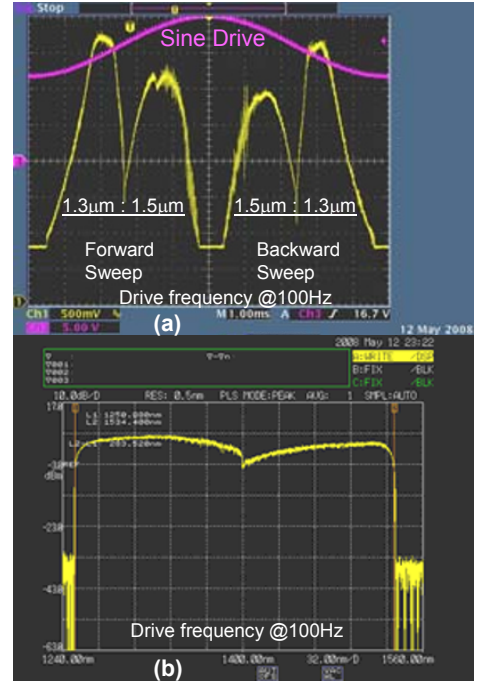

Fig. 5 Broadband CW swept laser performance at $100 \mathrm{~Hz}$ drive frequency, without fiber delay loop: (a) temporal swept laser profile and (b) swept optical spectrum showing a sweep range of about $283 \mathrm{~nm}$ and a discontinuity of about $5 \mathrm{~dB}$.

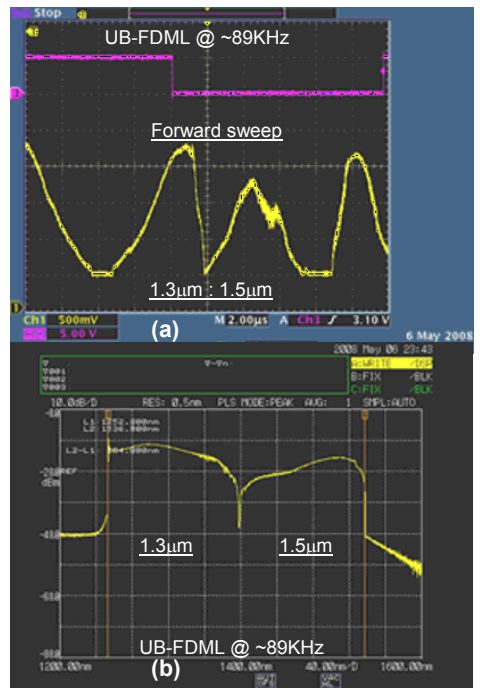

Fig. 6 Ultra broadband FDML laser performance at $89 \mathrm{kHz}$ sweep frequency: (a) temporal swept laser profile and (b) swept optical spectrum showing a sweep range of about $284 \mathrm{~nm}$, a wavelength gap of about $4 \mathrm{~nm}$, and a discontinuity of about $20 \mathrm{~dB}$.

\section{Imaging application}

We designed and developed a novel full-range Fourier domain optical coherence tomography (FD-OCT) system referring to dual-detection full-range FD-OCT (DD-FDOCT) that enables instantaneous retrieval of quadrature components of complex interferometric signal [12]. The DDFDOCT system enables full range imaging without 
loss of speed. Importantly, it is less sensitive to phase error generated by involuntary movements of subject in comparison to other established full range OCT systems because it uses two signals with a phase difference of $\pi / 2$ obtained simultaneously from two detecting arms to remove mirror images at all depths. This developed FD-OCT system benefits from the broad bandwidth $(158 \mathrm{~nm})$ of single-band FDML laser so that it enables $7 \mu \mathrm{m}$ axial resolution in air (wider than theoretical value due mainly to non-Gaussian spectrum), which is the highest axial resolution achieved among swept source based OCT at $1300 \mathrm{~nm}$ using a single SOA. The DD-FDOCT was applied to an in vivo human finger. We achieved a full range OCT image shown in Fig. 7 that consisted of 500 axial (A)-scans and 500 points per A-scan. In Fig. 7(a) the image using only a single detector shows the folded artifact image, while the artifact-free image generated in DD-FDOCT system is shown in Fig. 7(b). Both images are $2 \mathrm{~mm}$ by $2 \mathrm{~mm}$ in cross section.

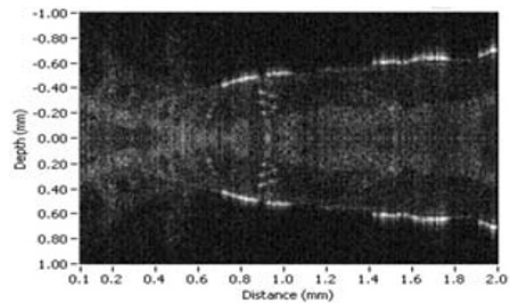

(a)

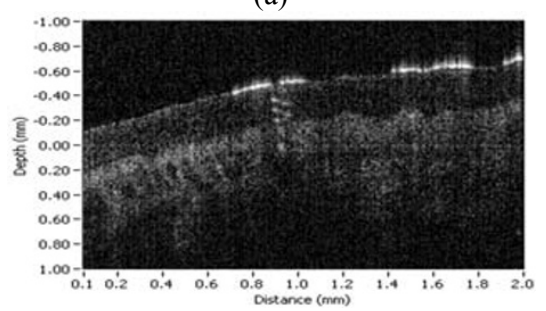

(b)

Fig. 7 Biological imaging (in vivo human finger): (a) real image and mirror image overlapped OCT image using a conventional FD OCT system and (b) mirror image removed OCT image using the DD-FDOCT system.

Moreover, the application of FDML laser for phase-resolved Doppler OCT was presented [13, 14]. The system exhibited high Doppler phase stability and allowed for flow monitoring over wide range of detectable flow velocity. The capability of DD-FD-OCT for phase-resolved Doppler imaging was investigated [13]. One of the advantages of DD-FD-OCT to Doppler imaging is that the full-range signal is achieved without manipulation of phase relation between consecutive axial lines. Therefore, the phase information of full-range signal acquired by DD-FD-OCT is almost identical to that acquired by conventional FD-OCT. Hence, it can be used for Doppler detection. Figure 8 shows Doppler imaging of an African frog tadpole by using full-range DD-FD-OCT system. Intensity images of the tadpole captured at a location close to heart chamber are shown in Figs. 8(a) and 8(b) for conventional FD-OCT and full-range DD-FD-OCT, respectively, processed from the same set of acquired spectra. Furthermore, Figs. 8(c) and 8(e) are zoom-in versions of the intensity images at the region indicated by the white dash box in Fig. 8(b) that contains one of the main blood vessels connected to heart chamber. The flow activity can be observed from Doppler images (Figs. 8(d) and 8(f)) where the mirror Doppler is completely suppressed in full-range mode (Fig. 8(f)) as compared with that process in conventional mode (Fig. 8(d)).
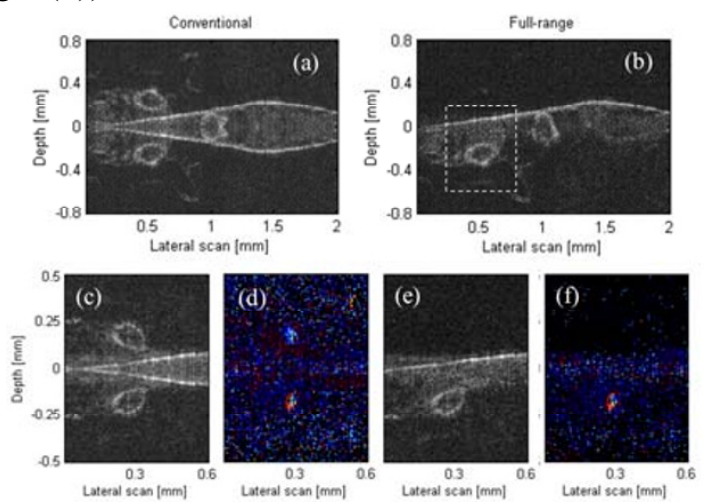

Fig. 8 Doppler images of an African frog tadpole: (a) and (b) are intensity images taken by conventional FD-OCT and DD-FD-OCT, respectively, (c) and (e) are zoom-in of (a) and (b) within the area marked by white dash box in (b), (d) and (f) are Doppler images corresponding with structural images in (c) and (e), respectively. 


\section{Conclusions}

We have demonstrated a FDML swept laser exhibiting a wide FWHM sweep range of about $158 \mathrm{~nm}$ at $89.2 \mathrm{kHz}$ sweep frequency in $1.3 \mu \mathrm{m}$ band by using a single SOA in an optimized low-loss ring cavity. To achieve broadband FDML operation, we designed a parallel SOA configuration to accommodate adjacent spectral windows and incorporate broadband optical components in common path. Indeed, a broadband spectrum at about $89 \mathrm{kHz}$ sweep frequency was generated over a sweep range from $1252.8 \mathrm{~nm}$ to $1396.8 \mathrm{~nm}$ and from $1400 \mathrm{~nm}$ to $1536.8 \mathrm{~nm}$, with an average output power of $3.5 \mathrm{~mW}$. We expect that $4 \mathrm{~nm}$ gap near $1400 \mathrm{~nm}$ wavelength region can be bridged by reducing cavity loss and by a custom fabricated $1.5 \mu \mathrm{m}$ SOA with a blue-shifted spectrum to boost the gain over WDM crossover region. Such high-speed broadband FDML lasers can significantly enhance the applications in high-resolution imaging and dynamic spectroscopy.

In the application of FDML lasers to high-speed and high-resolution in vivo biomedical imaging, a novel dual-detection full-range Fourier domain optical coherence tomography system is developed which provides $7 \mu \mathrm{m}$ axial resolution (in air) at about $90 \mathrm{kHz}$ A-scan rate for mirror-image resolved Doppler imaging in a human finger and an African frog tadpole.

\section{Acknowledgement}

This work was supported in part by the Florida I4 Corridor, the New York State Foundation for Science, Technology, and Innovation (NYSTAR), the Royal Thai Government, and the Photonics Technology Access Program (PTAP) sponsored by the Defense Advanced Research Projects Agency and National Science Foundation (DARPA \& NSF).

\section{References}

[1] M. A. Choma, K. Hsu, and J. A. Izat, "Swept source optical coherence tomography using an all-fiber $1300 \mathrm{~nm}$ fiber ring swept laser source," J. Bio-Medical Optics, vol. 10, no. 4, pp. 044009(1-6), 2005.

[2] R. Huber, M. Wojtkowski, K. Taira, J. G. Fujimoto, and K. Hsu, "Amplified, frequency swept lasers for frequency domain reflectometry and OCT imaging: Design and Scaling Principles," Optics Express, vol. 13, no. 9, pp. 3513-3528, 2005.

[3] J. Zhang, Q. Wang, B. Rao, Z. Chen, and K. Hsu, "Swept laser source at 1 micron for Fourier domain optical coherence tomography," Appl. Physics Lett., vol. 89, no. 7, pp. 073901(1-3), 2006.

[4] L. A. Kranendonk, X. An, A. W. Caswell, R. E. Herold, S. T. Sanders, R. Huber, J. G. Fujimoto, Y. Okura, and Y. Urata, "High speed engine gas thermometry by Fourier-domain mode-locked laser absorption spectroscopy," Optics Express, vol. 15, no. 23, pp. 15115-15128, 2007.

[5] K. Hsu, T. Haber, J. Mock, J. Volcy, and T. W. Graver, "High-speed swept-laser interrogation system for vibration monitoring," Structural Health Monitoring, Lancaster, PA: DEStech Publications, 2003, pp. 1043-1050.

[6] R. Huber, M. Wojtkowski, and J. G. Fujimoto, "Fourier Domain Mode Locking (FDML): A new laser operating regime and applications for optical coherence tomography," Optics Express, vol. 14, no. 8, pp. 3225-3237, 2006.

[7] R. Huber, D. C. Adler, V. J. Srinivasan, and J. G. Fujimoto, "Fourier domain mode locking at $1050 \mathrm{~nm}$ for ultra-high-speed optical coherence tomography of the human retina at 236,000 axial scans per second," Optics Letters, vol. 32, no. 14, pp. 2049-2051, 2007.

[8] D. C. Adler, S. W. Huang, R. Huber, and J. G. Fujimoto, "Photothermal detection of gold nanoparticles using phase-sensitive optical coherence tomography," Optics Express, vol. 16, no. 7, pp. 4376-4393, 2008.

[9] A. Bilenca, S. H. Yun, G. J. Tearney, and B. E. Bouma, "Numerical study of wavelength-swept semiconductor ring lasers: the role of refractive index nonlinearities in semiconductor optical amplifiers and implications for biomedical imaging applications," Optics Letters, vol. 31, no. 6, pp. 760-762, 2006.

[10] W. Y. Oh, S. H. Yun, G. J. Tearney, and B. E. Bouma, "Wide tuning range wavelength-swept laser with two semiconductor optical amplifiers," IEEE Photon. Technol. Lett., vol. 17, no. 3, pp. 678-680, 2005.

[11] M. Y. Jeon, J. Zhang, Q. Wang, and Z. Chen, "High-speed and wide bandwidth Fourier domain mode-locked wavelength swept laser with multiple SOAs," Optics Express, vol. 16, no. 4, pp. 25472554, 2008.

[12] K. Lee, P. Meemon, W. Dallas, K. Hsu, and J. P. Rolland, "Dual detection full range frequency domain optical coherence tomography," Opt. Lett., vol. 35, no. 7, pp. 1058-1060, 2010.

[13] P. Meemon, K. S. Lee, and J. P. Rolland, "Doppler imaging with dual-detection full-range frequency domain optical coherence tomography," Biomed. Opt. Express, vol. 1, no. 2, pp. 537-552, 2010.

[14] P. Meemon and J. P. Rolland, "Swept-source based, single-shot, multi-detectable velocity range Doppler optical coherence tomography," Biomed. Opt. Express, vol. 1, no. 3, pp. 955-966, 2010. 